\title{
Consumo de refrigerantes entre escolares de séries iniciais da cidade de Pelotas, Rio Grande do Sul
}

\author{
Consumption of soft drinks among students from early grades in the city \\ of Pelotas, Rio Grande do Sul \\ Thaynã Ramos Flores ${ }^{1}$, Carla Ribeiro Ciochetto ${ }^{2}$, Bruno Pereira Nunes ${ }^{3}$, \\ Maria de Fátima Alves Vieira ${ }^{4}$
}

\section{RESUMO}

Objetivo: Descrever o consumo diário de refrigerantes entre escolares do $1^{\circ}$ ao $4^{\circ}$ ano da rede municipal de ensino fundamental da cidade de Pelotas, Rio Grande do Sul.

Materiais e Métodos: Foi realizado um estudo transversal, de abordagem quantitativa, com 356 escolares do $1^{\circ}$ ao $4^{\circ}$ ano matriculados em escolas da zona urbana do município, no ano de 2011. O desfecho deste estudo foi o consumo diário de refrigerantes, avaliado através do Formulário de Marcadores de Consumo Alimentar proposto pelo Sistema de Vigilância Alimentar e Nutricional. A frequência de consumo foi descrita segundo gênero, idade, escolaridade materna, nível socioeconômico e estado nutricional do escolar. Foram consideradas as prevalências e os seus respectivos intervalos de confiança de 95\%, adotando-se o nível de significância estatística de 5\%.

Resultados: Dos 411 escolares considerados elegíveis para o estudo, foram avaliados 356, sendo de $13,4 \%$ as perdas e recusas. A prevalência de consumo diário de refrigerantes foi de $21,6 \%$. Não foram encontradas diferenças estatisticamente significativas de consumo diário de refrigerantes entre os sexos, faixa etária, classes de bens de consumo e estado nutricional.

Conclusão: Os escolares da rede municipal de ensino fundamental de Pelotas apresentaram elevada prevalência de consumo diário de refrigerantes. Diante esse quadro, ressalta-se a importância da educação nutricional no ambiente escolar.

Palavras-chave: consumo alimentar; hábitos alimentares; criança; refrigerantes.

\begin{abstract}
Objective: To describe the daily consumption of soft drinks among students from the 1st to the 4th year from public primary schools in the city of Pelotas, Rio Grande do Sul.

Materials and Methods: We conducted a cross-sectional study, with a quantitative approach, including 356 students from the 1st to the 4th year in the urban area of the city, in 2011. The outcome of this study was the daily consumption of soft drinks as was evaluated trough the Form Labels Food Consumption System proposed by the Food and Nutrition Surveillance. The frequency of consumption was described according to gender, age, maternal education, socioeconomic level and students' nutritional state. We analyzed the prevalence and its $95 \%$ confidence intervals, adopting the significance level of $5 \%$.

Results: Of the 411 students eligible for the study, 356 were evaluated, with $13.4 \%$ of losses and refusals. The prevalence of daily consumption of soft drinks was $21.6 \%$. There were no statistically significant differences in daily consumption of soft drinks between sexes, age, classes of consumer goods and nutritional state.

Conclusion: The students of the public primary schools of Pelotas showed a high prevalence of daily consumption of soft drinks. Therefore, we emphasize the importance of nutritional education in schools.
\end{abstract}

Keywords: food consumption; food habits; child; soft drinks.

\footnotetext{
${ }^{1}$ Acadêmica do Curso de Nutrição, Universidade Federal de Pelotas.

${ }^{2}$ Nutricionista. Mestre em Nutrição e Alimentos, Universidade Federal de Pelotas.

${ }^{3}$ Enfermeiro. Mestrando do Programa de Pós-Graduação em Epidemiologia, Universidade Federal de Pelotas.

${ }^{4}$ Nutricionista. Doutora em Ciências pela Universidade Federal de São Paulo (UNIFESP). Professora do Departamento de Nutrição, Universidade Federal de Pelotas.
} 


\section{INTRODUÇÃo}

A obesidade é uma epidemia mundial e mostra-se associada ao aumento das doenças crônicas não transmissíveis (DCNT) ${ }^{1}$. Estudos sugerem que essas doenças estão aumentando devido à transição nutricional e às constantes mudanças que estão ocorrendo nos hábitos alimentares ${ }^{2,3}$.

Nos últimos anos houve uma incorporação de bebidas e alimentos industrializados nos hábitos alimentares de crianças, adolescentes e adultos. Entre esses produtos industrializados, destaca-se o refrigerante ${ }^{4}$. Segundo alguns autores, o consumo de refrigerantes caracterizase como uma prática alimentar inadequada ${ }^{5,6}$, e evidências sugerem que 0 alto consumo dessa bebida promove ganho excessivo de peso ${ }^{7}$.

Estudo realizado nos Estados Unidos indica que houve um aumento em $500 \%$ no consumo de refrigerantes nos últimos 50 anos. Ainda que o refrigerante seja uma fonte desnecessária de açúcar adicionado à dieta de crianças e adolescentes, seu consumo diário ainda é elevado nessas faixas etárias ${ }^{8}$.

No Brasil, dados publicados na Pesquisa de Orçamentos Familiares (POF's) de 2002-2003 evidenciaram que a disponibilidade domiciliar de refrigerantes aumentou $400 \%$ quando comparado com a década de 70 . A pesquisa mostrou que a disponibilidade da ingestão de refrigerantes cresceu conforme a renda, sendo cinco vezes maior nas classes de renda mais elevada quando comparados as de menor renda 9 . Dados recentes da POF de 2008-2009, que analisou o consumo alimentar da população, evidenciaram que o aumento na ingestão de refrigerantes apresentou relação direta com a renda ${ }^{10}$.

Atualmente não existem recomendações que limitem a ingestão de refrigerantes para o público infantil. $O$ Guia Alimentar Brasileiro propõe o consumo de uma maior variedade de alimentos "in natura", que incluam pães, cereais, frutas e hortaliças, utilizando-se sal e açúcar com moderação. Além disso, recomenda redução no consumo de bebidas processadas com alta concentração de açúcar, categoria de alimentos que inclui os refrigerantes ${ }^{11}$. O refrigerante é uma bebida à base de açúcar, desprovida de valor nutricional, e, portanto, pode contribuir para o consumo excessivo de calorias vazias. Além disso, pode substituir alimentos com valor nutricional que atuam no crescimento e desenvolvimento durante a infância e adolescência ${ }^{12,13}$. Neste sentido, o presente estudo teve como objetivo descrever o consumo diário de refrigerantes entre escolares de $1^{\circ}$ ao $4^{\circ}$ ano da rede pública municipal de ensino fundamental, da cidade de Pelotas, Rio Grande do Sul.

\section{MATERIAIS E MÉTODOS}

Foi realizado um estudo transversal de abordagem quantitativa, com escolares do $1^{\circ}$ ao $4^{\circ}$ ano da rede pública municipal de ensino fundamental da cidade de Pelotas, Rio Grande do Sul. A coleta de dados e das medidas antropométricas foi realizada entre os meses de março a junho do ano de 2011. O município de Pelotas possui 40 escolas com oferta de ensino fundamental na zona urbana da cidade. Seis dessas escolas foram escolhidas através de um sorteio aleatório e, após essa seleção, foi realizado um segundo sorteio para eleger uma turma por escola de $1^{\circ}, 2^{\circ}, 3^{\circ}$ ou $4^{\circ}$ ano. Utilizou-se como critério de exclusão as instituições de ensino que apresentavam turmas multiseriadas e que já haviam participado de projetos da Faculdade de Nutrição da Universidade Federal de Pelotas.

As informações sobre as condições sociodemográficas e de consumo alimentar foram coletadas através de um questionário aplicado à mãe ou ao responsável pelo escolar, com duração máxima de 30 minutos, em visita domiciliar. Todos os dados foram coletados por entrevistadoras previamente treinadas e capacitadas para os instrumentos utilizados.

Para a avaliação das práticas alimentares das crianças, foi utilizado o Formulário de Marcadores de Consumo Alimentar proposto pelo Sistema de Vigilância Alimentar e Nutricional (SISVAN) do Ministério da Saúde do Brasil $^{14}$. O referido instrumento tem como objetivo avaliar a frequência de consumo de 10 grupos de alimentos específicos nos últimos sete dias que antecedem a entrevista, e são assim descritos: saladas cruas (alface, tomate, cenoura, pepino e outros), legumes e verduras cozidas (excluindo batata $e$ mandioca), frutas frescas e salada de fruta, feijão, leite 
ou iogurte, batata frita e salgados fritos, hambúrguer e embutidos, bolachas/biscoito doces ou recheados e chocolates, refrigerantes (excluindo os light ou diet).

O consumo de refrigerantes constitui o desfecho do estudo e foi avaliado através do instrumento do SISVAN $^{14}$, o qual abordou a frequência de consumo no decorrer dos sete dias que antecederam a entrevista. Para descrição do desfecho foi utilizado o consumo diário (sete dias) de refrigerantes.

Foram coletadas informações demográficas (gênero e idade), faixa etária, sendo de 5 a 9 anos de idade (crianças) e com idade igual ou maior que 10 anos (adolescentes). As variáveis relacionadas à condição socioeconômica foram: escolaridade materna em anos completos ( $\leq 4 ; 5-8 ; \geq 9$ anos) e a classe de consumo de bens segundo a metodologia da Associação Brasileira de Empresas de Pesquisas ${ }^{15}$, que classifica as famílias segundo posse de bens em 5 grupos (A/B/C/D/E). Devido ao reduzido número de escolares pertencentes à classe socioeconômica A e $E$, a classe A foi agrupada com a $B(A+B)$ e a classe $E$ foi agrupada com a $D$ $(\mathrm{D}+\mathrm{E})$.

A avaliação do estado nutricional foi realizada através de medidas antropométricas utilizando dados de peso e de altura segundo critérios de Lohman et al. ${ }^{16}$, essas medidas foram coletadas no ambiente escolar no mesmo período em que acontecia a coleta de dados em visita domiciliar. Os equipamentos utilizados para a coleta das medidas antropométricas foram: balança solar portátil da marca Tanita® (modelo 1631, Japão) para obtenção do peso, com capacidade para 150 quilogramas e precisão de 200 gramas e fita métrica inextensível para aferição da altura, de $150 \mathrm{~cm}$ de comprimento afixada de forma invertida com fitas adesivas em parede plana e sem rodapé estando posicionada a $50 \mathrm{~cm}$ do chão. As medidas de peso e altura foram aferidas duas vezes e os escolares estavam descalços, vestindo roupas leves e sem adereços no cabelo. A análise dos dados foi realizada no programa Anthro-Plus $\mathrm{WHO}^{17}$, adotando como indicador do estado nutricional o Índice de Massa Corporal (IMC) por idade, em escores-z, e como referência as curvas da Organização Mundial da Saúde para crianças e adolescentes de 5 a 19 anos. Para fins de análise do estado nutricional, foram considerados com excesso de peso os escolares que apresentaram valores acima de um escore-z, e sem excesso de peso aqueles com escore-z menor ou igual a $\mathrm{um}^{18}$.

O controle de qualidade dos dados foi feito pelo coordenador do trabalho, através de entrevista telefônica com $10 \%$ da amostra. Os dados foram duplamente digitados no programa Epi Info versão 6.04 (Centers for Disease Control and Prevention, Atlanta, Estados Unidos). Foram consideradas as prevalências de consumo de refrigerantes e os seus respectivos intervalos de confiança de 95\%, adotando-se o nível de significância estatística de $5 \%$. Utilizou-se o teste quiquadrado de heterogeneidade para verificar as associações. As análises foram realizadas através do programa estatístico Stata versão 11.0 (Stata Corp., College Station, Estados Unidos).

O projeto obteve aprovação do Comitê de Ética em Pesquisa da Faculdade de Medicina da Universidade Federal de Pelotas no ano de 2011 sob o número de protocolo 11/11, e autorização da Secretaria Municipal de Educação do Município e ainda dos pais ou responsáveis pelos escolares, através de assinatura do termo de consentimento livre e esclarecido.

\section{RESUTADOS}

Dos 411 escolares considerados elegíveis para o estudo obteve-se amostra de 356 avaliados, houve $13,4 \%$ de perdas e recusas sendo que a maioria (67\%) refere-se ao gênero masculino e a metade das perdas ocorreu em uma escola devido a sua política de fornecimento de endereços dos escolares, o que impediu o acesso aos pais ou responsáveis para realização da entrevista.

$\mathrm{Na}$ tabela 1 estão descritos os dados referentes às características sociodemográficas e estado nutricional da amostra. Mais da metade dos alunos eram do sexo masculino $(53,9 \%)$, com faixa etária entre 5 e 9 anos de idade $(80,3 \%)$ e pertencentes a classe $C$ de consumo de bens $(63,2 \%)$. Em relação à escolaridade materna, a maioria das mães encontrava-se no grupo de 9 ou mais anos completos de estudos (43,2\%). Quanto à avaliação do estado nutricional $(n=343)$, houve perdas de $3,7 \%$ dos escolares participantes do estudo, por não estarem 
TABELA 1 - Características sociodemográficas e de estado nutricional dos escolares de $1^{\circ}$ ao $4^{\circ}$ ano do ensino fundamental $(n=356)$.

\begin{tabular}{lcc}
\hline Variáveis & $\mathbf{N}$ & $\%$ \\
\hline Gênero & & \\
$\quad$ Masculino & 192 & 53,9 \\
$\quad$ Feminino & 164 & 46,1 \\
$\quad$ Idade (em anos) & & \\
$\quad 5-9$ & 286 & 80,3 \\
$\quad 10-15$ & 70 & 19,6 \\
Nível socioeconômico & & \\
A - B & 83 & 23,3 \\
C & 225 & 63,2 \\
$\quad$ D - E & 48 & 13,4 \\
Escolaridade materna (em anos & & \\
completos) & & \\
$\quad \leq 4$ & 65 & 18,3 \\
$5-8$ & 137 & 38,5 \\
$\quad \geq 9$ & 154 & 43,2 \\
Estado nutricional & \\
$\quad$ Com excesso de peso & & \\
Sem excesso de peso & 150 & 43,7 \\
\hline
\end{tabular}

${ }^{a}$ valores ignorados para variável excesso de peso $(n=13)$.

presentes na escola no dia da coleta das medidas antropométricas. Mais da metade dos alunos foram classificados sem excesso de peso (56,3\%) de acordo com o indicador IMC para idade, e a prevalência de escolares com excesso de peso foi de $43,7 \%$.

A tabela 2 apresenta a distribuição da frequência semanal, em dias, do consumo total de refrigerantes entre os escolares de acordo com as variáveis independentes. O consumo diário de refrigerantes foi mais freqüente no gênero feminino $(22,6 \%)$, nos escolares com idade entre 5-9 anos (23,4\%), entre aqueles indivíduos que pertenciam à classe $\mathrm{C}$ de consumo de bens $(24,4 \%)$ e em filhos de mães que estudaram de 5 - 8 anos completos (24,1\%). Quanto ao estado nutricional, a maior prevalência de consumo diário foi observada em escolares sem excesso de peso (23,8\%). Em geral, 14,3\% não ingeriram essa bebida na semana que antecedeu a pesquisa e a prevalência de consumo diário foi observada em $21,6 \%$ dos escolares.

A tabela 3 apresenta a associação entre a freqüência de consumo diário de refrigerantes segundo as variáveis independentes e seus respectivos intervalos de confiança. Não foram observadas associações estatisticamente significativas entre o consumo diário de refrigerantes e as variáveis independentes.

\section{DISCUSSÃO}

Os hábitos alimentares saudáveis são fundamentais na prevenção da obesidade e de doenças crônicodegenerativas, e os inquéritos de consumo alimentar desempenham papel importante na identificação do padrão alimentar de crianças, adolescentes e adultos $^{3,19}$. O presente estudo avaliou o consumo de refrigerantes entre escolares, mostrando que de forma geral o consumo diário apresentou-se elevado entre os alunos matriculados nas quatro primeiras séries do ensino fundamental na cidade de Pelotas.

Segundo Levy-Costa et al. ${ }^{20}$, há uma tendência de crescimento do consumo de refrigerantes no Brasil. Neste estudo, encontrou-se elevada prevalência de consumo diário de refrigerantes, sendo semelhante ao identificado em alguns estudos brasileiros ${ }^{21,22,23}$ e em uma pesquisa da Organização Mundial de Saúde ${ }^{24}$. Além disso, cerca de $86 \%$ dos escolares do presente estudo consumiram refrigerantes no decorrer dos sete dias que antecederam a entrevista. Estes resultados sugerem que 0 hábito de ingerir refrigerantes está fortemente inserido na vida dos escolares avaliados.

Entre as seis escolas avaliadas neste estudo, duas delas possuíam cantinas e destas apenas uma comercializava refrigerantes. A oferta de refrigerantes está diretamente relacionada ao aumento do consumo dessa bebida. Ainda, é importante salientar que a influência exercida pela família e pela escola é fundamental para a formação e manutenção dos hábitos alimentares de crianças e adolescentes ${ }^{25}$. Muitas instituições de ensino facilitam o acesso ao refrigerante através da comercialização de alimentos calóricos e bebidas açucaradas. Segundo estudos realizados nas cidades de Ribeirão Preto e no Rio de Janeiro, o refrigerante está entre os produtos mais ofertados às crianças nas escolas ${ }^{26}$. Estudo realizado em Porto Alegre observou que mais de $90 \%$ das escolas avaliadas comercializavam refrigerantes ${ }^{27}$. No estado de Santa Catarina, importantes mudanças ocorreram com a aprovação da Lei de Regulamentação das Cantinas ${ }^{28}$, a qual proíbe a comercialização de refrigerantes e de outros alimentos calóricos no ambiente escolar. 
TABELA 2 - Distribuição da frequência de consumo de refrigerantes segundo variáveis sociodemográficas e de estado nutricional em escolares do $1^{\circ}$ ao $4^{\circ}$ ano do ensino fundamental $(n=356)$.

\begin{tabular}{|c|c|c|c|c|c|c|c|c|}
\hline \multirow{2}{*}{ Variáveis } & \multicolumn{8}{|c|}{ Frequência semanal (em dias) } \\
\hline & 0 & 1 & 2 & 3 & 4 & 5 & 6 & 7 \\
\hline \multicolumn{9}{|l|}{ Gênero } \\
\hline Masculino & 17,7 & 15,6 & 18,2 & 13,5 & 9,4 & 1,6 & 3,1 & 20,8 \\
\hline Feminino & 10,4 & 24,4 & 17,1 & 11,6 & 6,7 & 3,0 & 4,3 & 22,6 \\
\hline \multicolumn{9}{|l|}{ Idade (em anos) } \\
\hline $5-9$ & 12,9 & 20,3 & 16,8 & 12,9 & 7,3 & 2,4 & 3,8 & 23,4 \\
\hline $10-15$ & 20,0 & 17,1 & 21,4 & 11,4 & 11,5 & 1,4 & 2,9 & 14,3 \\
\hline \multicolumn{9}{|l|}{ Nível socioeconômico } \\
\hline$A-B$ & 16,9 & 14,4 & 19,3 & 14,5 & 10,8 & 3,6 & 1,2 & 19,3 \\
\hline C & 10,2 & 21,8 & 17,8 & 12,0 & 8,0 & 2,2 & 3,6 & 24,4 \\
\hline$D-E$ & 29,2 & 18,7 & 14,6 & 12,5 & 4,2 & 0 & 8,3 & 12,5 \\
\hline \multicolumn{9}{|c|}{ Escolaridade materna (em anos completos) } \\
\hline$\leq 4$ & 23,1 & 12,3 & 15,4 & 12,3 & 12,3 & 1,5 & 4,6 & 18,5 \\
\hline $5-8$ & 10,2 & 22,6 & 17,5 & 11,7 & 4,4 & 3,6 & 5,8 & 24,1 \\
\hline$\geq 9$ & 14,3 & 20,1 & 18,8 & 13,7 & 9,7 & 1,3 & 1,3 & 20,8 \\
\hline \multicolumn{9}{|l|}{ Estado nutricional $^{\mathrm{a}}$} \\
\hline Com excesso de peso & 18,0 & 19,3 & 18,7 & 10,7 & 8,7 & 2,7 & 3,3 & 18,7 \\
\hline Sem excesso de peso & 11,4 & 20,1 & 16,1 & 14,5 & 7,8 & 2,1 & 4,1 & 23,8 \\
\hline Total do consumo de refrigerantes & 14,3 & 19,7 & 17,7 & 12,6 & 8,2 & 2,3 & 3,7 & 21,6 \\
\hline
\end{tabular}

${ }^{a}$ valores ignorados para variável excesso de peso $(n=13)$.

Entretanto, a fiscalização das cantinas é necessária para a consolidação da lei $^{29}$.

De acordo com o estudo de Levy et al. ${ }^{30}$ o consumo de refrigerantes foi mais freqüente entre 0 gênero masculino. Entretanto, os resultados do presente estudo não apresentaram diferenças significativas entre os sexos com relação ao consumo diário de refrigerantes, semelhante aos obtidos por Nogueira e Schieri ${ }^{22}$, ao estudarem escolares de Niterói.

Embora alguns estudos evidenciem maior proporção de consumo de refrigerantes com o aumento da faixa etária $^{22,31}$, neste estudo, diferenças relacionadas à idade dos escolares não foram observadas. É possível que o consumo de refrigerantes cresça de acordo com a faixa etária em consequência da maior autonomia que os adolescentes possuem ao realizar as suas escolhas alimentares $^{32}$. Ainda, dados presentes na literatura ressaltam que bebidas mais nutritivas acabam sendo substituídas pelo refrigerante com 0 aumento da idade $^{33,34,35}$.

Nesta pesquisa não se encontrou associação entre consumo diário de refrigerantes e nível socioeconômico sendo o mesmo observado em estudo multicêntrico sobre o consumo alimentar, realizado em Campinas, Rio de Janeiro, Goiânia, Ouro Preto e Curitiba, no qual o consumo de refrigerantes foi semelhante em todas as faixas de renda ${ }^{36}$. No presente estudo, parece que o nível socioeconômico não influenciou o consumo de refrigerantes, atingindo igualmente todas as classes sociais. Um fator que pode facilitar o acesso a essas bebidas é o custo. Atualmente os refrigerantes estão com preços mais baixos e vem sendo vendidos em embalagens econômicas, facilitando o acesso, mesmo para indivíduos com menor poder aquisitivo. Porém, de acordo com outro estudo brasileiro foi observado maior consumo de refrigerantes entre crianças que pertenciam a famílias com melhores condições socioeconômicas ${ }^{37}$. Segundo o autor, crianças que pertencem a um nível socioeconômico mais favorecido possuem maior acesso aos alimentos que contém quantidades significativas de calorias vazias $^{38,39}$, como os refrigerantes.

A escolaridade materna pode ser considerada um fator importante na escolha de alimentos saudáveis, uma vez que os pais e familiares são os primeiros a contribuir para a formação dos hábitos alimentares das crianças $^{40}$. Na presente análise, não foram observadas 
TABELA 3 - Prevalência de consumo diário segundo variáveis sociodemográficas e de estado nutricional em escolares do $1^{\circ}$ ao $4^{\circ}$ ano do ensino fundamental $(n=356)$.

\begin{tabular}{|c|c|c|}
\hline Variáveis & Prevalência de consumo diário $\left(\mathbf{I C}_{95 \%}\right)$ & $\boldsymbol{P}$ \\
\hline Gênero & & 0,693 \\
\hline Masculino & $20,8(15,0-26,6)$ & \\
\hline Feminino & $22,6(16,1-29,0)$ & \\
\hline Idade (em anos) & & 0,096 \\
\hline $5-9$ & $23,4(18,5-28,4)$ & \\
\hline $10-15$ & $14,3(5,9-22,7)$ & \\
\hline Nível socioeconômico & & 0,159 \\
\hline$A-B$ & $19,3(10,6-27,9)$ & \\
\hline $\mathrm{C}$ & $24,4(18,8-30,1)$ & \\
\hline$D-E$ & $12,5(2,8-22,2)$ & \\
\hline Escolaridade materna (em anos completos) & & 0,625 \\
\hline$\leq 4$ & $18,5(8,0-28,1)$ & \\
\hline $5-8$ & $24,1(16,8-31,3)$ & \\
\hline$\geq 9$ & $20,8(14,3-27,2)$ & \\
\hline Estado nutricional $^{a}$ & & 0,248 \\
\hline Com excesso de peso & $18,7(12,3-25,0)$ & \\
\hline Sem excesso de peso & $23,8(17,8-29,9)$ & \\
\hline
\end{tabular}

diferenças relacionadas à escolaridade materna, ao contrário dos resultados relatados por Toloni et al. ${ }^{41}$ que ao estudar a introdução de alimentos industrializados entre crianças, verificou que quanto maior o grau de escolaridade materno, menor o consumo de alimentos e bebidas que contenham em sua composição alto teor de gordura, açúcar e sal.

Diferenças com significância estatística no consumo diário de refrigerantes associadas ao estado nutricional não foram identificadas, semelhante ao que foi observado no estudo de Nogueira e Schieri ${ }^{22}$. No entanto, outros estudos brasileiros identificaram que o consumo de refrigerantes apresentou associação importante entre escolares que tinham excesso de peso e obesidade ${ }^{42,43}$. Devido ao delineamento transversal do presente estudo, o qual realiza a mensuração do consumo de refrigerantes e da medida antropométrica ao mesmo tempo, a inferência sobre a causalidade do desfecho fica prejudicada, uma vez que o estado nutricional pode influenciar nos hábitos alimentares das pessoas $^{44}$.

O presente estudo avaliou apenas escolas municipais, entretanto a distribuição da amostra com relação às variáveis estudadas mostrou-se semelhante à distribuição observada na população brasileira em geral, por exemplo, com relação às classes de consumo, sexo e escolaridade materna. Em relação ao instrumento utilizado, cabe ressaltar que omissões quanto ao relato do consumo de refrigerantes podem ter ocorrido. Destaca-se como aspecto positivo o fato da amostra estudada representar os escolares do $1^{\circ}$ ao $4^{\circ}$ ano do ensino fundamental pertencentes à rede municipal de Pelotas, RS.

O consumo elevado de refrigerantes entre crianças e adolescentes é preocupante, uma vez que as DCNT possuem um período de latência para ocorrer, podendo surgir a médio ou a longo prazo ${ }^{45}$. Além disso, o público infantil é o principal alvo de propagandas indiscriminadas vinculadas a alimentos e bebidas açucaradas $^{46}$. Sendo assim, intervenções intersetoriais direcionadas a esse público para promoção da alimentação saudável são necessárias para o enfrentamento das $\mathrm{DCNT}^{47}$ uma vez que os hábitos fixados na infância têm maior probabilidade de permanecer na adolescência e na vida adulta ${ }^{29}$.

Os escolares da rede municipal de ensino fundamental de Pelotas apresentaram elevada prevalência de consumo diário de refrigerantes. Diante esse quadro, ressalta-se a importância da educação nutricional no ambiente escolar, como uma estratégia 
importante para a promoção de hábitos alimentares saudáveis visto que possibilita a inclusão de professores, profissionais da área de nutrição, pais, alunos e merendeiras (manipuladores de alimentos), garantindo maior êxito desta intervenção para a alimentação saudável das crianças e adolescentes.

\section{Agradecimentos}

Agradecemos às escolas e entrevistados que participaram do estudo, ao Centro Colaborador em Alimentação e Nutrição do Escolar da Universidade Federal do Rio Grande do Sul (CECANE- UFRGS), o qual foi o financiador do projeto, e à Faculdade de Nutrição da Universidade Federal de Pelotas.

\section{REFERÊNCIAS}

1. Schmidt MI, Duncan BB, Silva GA, Menezes AM, Monteiro CA, Barreto SM, Chor D, Menezes PR. Doenças crônicas não transmissíveis no Brasil: carga e desafios atuais. [monografia na internet]. Saúde no Brasil 4. Oxford: Elsevier; 2011. [capturado 2013 Fev 05]. Disponível em: http://download.thelancet.com/flatcontentassets/pdfs/brazil /brazilpor4.pdf. [Global Health Series. Saúde no Brasil; 4]

2. Batista Filho M, Rissini A. A transição nutricional no Brasil: tendências regionais e temporais. Cad Saúde Pública. 2003;19 Suppl 1:s181-s91.

3. Monteiro CA, Mondini L, Souza AL, Popkin B. Da desnutrição para a obesidade: a transição nutricional no Brasil. In: Monteiro CA, ed. Velhos e Novos Males da Saúde no Brasil: a evolução do país e suas doenças, $2^{\underline{a}}$ edição, São Paulo: Editora Hucitec; 2000. p.247-55.

4. Bleil SI. O padrão alimentar ocidental: considerações sobre a mudança de hábitos no Brasil. Cad Debate. 1998;6:1-25.

5. Carmo MB, Toral N, Silva MV, Slater B. Consumo de doces, refrigerantes e bebidas com adição de açúcar entre adolescentes da rede pública de ensino de Piracicaba, São Paulo, Brasil. Rev Bras Epidemiol. 2006;9(1):121-30.

6. Conceição SIO, Santos CJN, Silva AAM, Silva JS, Oliveira TC. Consumo alimentar de escolares das redes pública e privada do ensino em São Luís, Maranhão. Rev Nutr. 2010;23(6):993-1004.

7. World Health Organization. Diet, nutrition and the prevention of chronic diseases: report of a joint WHO/FAO expert consultation. Geneva: WHO; 2003. Technical Report Series 916. 160 p.

8. Ludwig DS, Peterson KE, Gortmaker SL. Relation between consumption of sugar-sweetened drinks and childhood obesity: a prospective, observational analysis. Lancet. 2001;357:505-8.

9. Instituto Brasileiro de Geografia e Estatística. Pesquisa de orçamentos familiares 2002-2003: primeiros resultados Brasil e grandes regiões. Rio de Janeiro: IBGE; 2004.

10. Instituto Brasileiro de Geografia e Estatística. Pesquisa de orçamentos familiares 2008-2009: análise do consumo alimentar pessoal no Brasil. Rio de Janeiro: IBGE; 2011.

11. BRASIL. Ministério da Saúde. Secretaria de Atenção à Saúde. Departamento de Atenção Básica. Coordenação Geral da Política de Alimentação e Nutrição. Guia alimentar para a população brasileira. Brasília: $\mathrm{O}$ Ministério; 2006.
12. Vartanian LR, Schwartz MB, Brownell KD. Effects of soft drink consumption on nutrition and health: a systematic review and meta-analysis. Am J Public Health 2007;97(4):667-75.

13. Grimm GC, Harnack L, Story M. Factors associated with soft drink consumption in school-aged children. J Am Diet Assoc 2004;104(8):1244-9.

14. BRASIL. Ministério da Saúde. Princípios e Diretrizes de uma política de segurança alimentar e nutricional. Protocolos do Sistema de Vigilância Alimentar e Nutricional - SISVAN na assistência à saúde. Brasília: O ministério; 2008. [Série B. Textos Básicos de Saúde].

15. Associação Brasileira de Empresas de Pesquisa. Critério de classificação econômica Brasil. [monografia na internet]. São Paulo: ABEP; 2009 [capturado em 2011 Ago 4]. Disponível em: http://www.abep.org/novo/ Content.aspx?ContentID=301

16. Lohman T, Roche A, Martorell R. Anthropometric standardization referece manual. Champaing: Human Kinetics Books; 1988.

17. World Health Organization. Growth reference data for 5-19 years, WHO reference 2007. [monografia na internet]. Geneva: WHO; [2007] [capturado 2011 Set 10]. Disponível em: http://www.who.int/growthref/en

18. de Onis M, Onyango AW, Borghi E, Siyam A, Nishida C, Siekmann J. Development of a WHO growth reference for school-aged children and adolescents. Bull World Health Organ. 2007;85(9):660-7.

19. Holanda LB, Barros Filho AA. Métodos aplicados em inquéritos alimentares. Rev Paul Pediatria. 2006;24(1):6270.

20. Levy-Costa RB, Sichieri R, Pontes NS, Monteiro CA. Disponibilidade domiciliar de alimentos no Brasil: distribuição e evolução (1974-2003). Rev Saúde Pública. 2005;39(4):530-40.

21. Carvalho AP, Oliveira VB, Santos LC. Hábitos alimentares e práticas de educação nutricional: atenção a crianças de uma escola municipal de Belo Horizonte, Minas Gerais. Pediatria. 2010;32(1):20-7.

22. Nogueira FAM, Sichieri R. Associação entre consumo de refrigerantes, sucos e leite, com o índice de massa corporal em escolares da rede pública de Niterói, Rio de Janeiro. Cad Saúde Pública. 2009;25(12):2715-24.

23. Malta DC, Sardinha LMV, Mendes I, Barreto SM, Giatti L, Castro IRR, Moura L, Dias AJR, Crespo C. Prevalência de fatores de risco e proteção de doenças crônicas não transmissíveis em adolescentes: resultados da Pesquisa Nacional de Saúde do Escolar (PeNSE), Brasil, 2009. Ciênc Saúde Coletiva. 2010;5 Suppl 2:3009-19.

24. Currie C, Gabhainn SN, Godeau E, Roberts C, Smith R, Currie D, Picket W, Richter M, Morgan A, Barnekow V. Inequalities young people's health: HBSC International Report from the 2005/2006 survey. [monografia na internet]. Copenhagen: WHO; c2008 [capturado $2012 \mathrm{Abr}$ 13]. Disponível em: http://www.euro.who.int/_data/assets/ pdf_file/0005/53852/E91416.pdf. [Health Policy for Children and Adolescents; 5]

25. Estima CCP, Philippi ST, Alvarenga MS. Fatores determinantes de consumo alimentar: por que os indivíduos comem o que comem? Rev Bras Nutr Clin 2009;24(4) 263-8.

26. BRASIL. Ministério da Saúde. Regulamentação da comercialização de alimentos em escolas no Brasil: experiências estaduais e municipais. [monografia na internet]. Brasília: Ministério da Saúde, 2007 [capturado 2012 Nov 26]. Disponível em: http://189.28.128.100/ nutricao/docs/geral/regula_comerc_alim_escolas_exper_e staduais_municipais.pdf.

27. Willhelm FF, Ruiz E, Oliveira AB. Cantina escolar: 
qualidade nutricional e adequação à legislação vigente. Rev HCPA. 2010;30(3):266-70.

28. Santa Catarina. Secretaria de Estado da Educação. Lei $n^{\circ}$ 12.061 , de 18 de dezembro de 2001. Dispõe sobre critérios de concessão de serviços de lanches e bebidas nas unidades educacionais, localizadas no Estado de Santa Catarina. Diário Oficial [do] Estado de Santa Catarina, Florianópolis, SC, n. 16.810, 20 dez 2001.

29. Fernandes OS, Bernardo CO, Campos RMMB, Vasconcelos FAG. Avaliação do efeito da educação nutricional na prevalência de sobrepeso/obesidade e no consumo alimentar de escolares do ensino fundamental. $J$ Pediatr. 2009;85(4):315-21.

30. Levy RB, Castro IRR, Cardoso LO, Tavares LF, Sardinha LMV, Gomes FS, Costa AWN. Consumo e comportamento alimentar entre adolescentes brasileiros: Pesquisa Nacional de Saúde do Escolar (PeNSE). Ciênc Saúde Coletiva. 2010;15(2):3085-97.

31. Dubois L, Farmer A, Girard M, Peterson K. Regular sugarsweetened beverage consumption between meals increases risk of overweight among preschool-aged children. J Am Diet Assoc. 2007;107(6):924-34.

32. Danelon MAS, Danelon MS, Silva MV. Serviços de alimentação destinados ao público escolar: análise da convivência do Programa de Alimentação Escolar e das cantinas. Segur Aliment Nutr. 2006;13(1):85-94

33. Harnack L, Stang J, Story M. Soft drink consumption among US children and adolescents: nutritional consequences. J Am Diet Assoc 1999;99:436-41.

34. Mrdjenovic G, Levitsky DA. Nutritional and energetic consequences of sweetened drink consumption in 6- to 13-year-old children. J Pediatr 2003;142:604-10.

35. Forshee RA, Storey ML. Total beverage consumption and beverage choices among children and adolescents. Int $\mathrm{J}$ Food Sci Nutr. 2003;54(4):297-307.

36. Galeazzi MAN, Domene SMA, Sichieri R. Estudo multicêntrico sobre consumo alimentar. Cad Debate 1997;nesp.

37. Drewnowski A, Monsivais P, Maillot M, Darmon N. Lownergy-density diets are associated with higher diet quality and higher diet costs in French adults. J Am Diet Assoc. 2007;107:1028-32.

38. Aquino RC, Philippi ST. Consumo infantil de alimentos industrializados e renda familiar na cidade de São Paulo. Rev Saúde Pública. 2002;36(6):655-60.

39. Leão LSCS, Araújo LMB. Prevalência de obesidade em escolares de Salvador, Bahia. Arq Bras Endocrinol Metab. 2003;47(2):151-7.

40. Wilhelm FA, Lima JHCA, Schirmer KF. Obesidade infantil e a família: educadores emocionais e nutricionais dos filhos. Psicol Argum 2007;25(49):143-154.

41. Toloni MHA, Longo-Silva G, Goulart RMM, Taddei JAAC. Introdução de alimentos industrializados e de alimentos de uso tradicional na dieta de crianças de creches públicas no município de São Paulo. Rev Nutr. 2011;24(1):61-70.

42. Lopes PCS, Prado SRLA, Colombo P. Fatores de risco associados à obesidade e sobrepeso em crianças em idade escolar. Rev Bras Enferm. 2010;63(1):73-8.

43. Polla SF, Scherer F. Perfil alimentar e nutricional de escolares da rede municipal de ensino de um município do interior do Rio Grande do Sul. Cad Saúde Colet. 2011;19(1):111-6.

44. Rombaldi AJ, Neutzling MB, Silva MC, Azevedo MR, Hallal PC. Fatores associados ao consumo regular de refrigerante não dietético em adultos de Pelotas, RS. Rev. Saúde Pública. 2011;45(2):382-390.

45. Guedes DP. Educação para a saúde mediante programa de educação física escolar. Motriz. 1999;5(1):10-5.

46. BRASIL. Ministério da Saúde. Secretaria de Atenção à Saúde. Departamento de Atenção Básica. Obesidade. Brasília: O Ministério; 2006. [Caderno de Atenção Básica № 12. Série A. Normas e Manuais Técnicos]

47. Vieira MFA, Araújo CLP, Hallal PC, Madruga SW, Neutzling MB, Matijasevich A, Leal CMA, Menezes AMB. Estado nutricional de escolares de $1^{\underline{a}}$ a $4^{\underline{a}}$ séries do Ensino Fundamental das escolas urbanas da cidade de Pelotas, Rio Grande do Sul, Brasil. Cad Saúde Pública. 2008;24(7):1667-1674.

\section{Endereço para correspondência}

Thaynã Ramos Flores

Rua Gomes Carneiro $n^{\circ} 1$

Pelotas/RS - CEP 96010-610

Telefone: +55 5339211259

E-mail: thaynaramosflores@yahoo.com.br 